\title{
ON THE ALGEBRAIC INDEPENDENCE OF SYMMETRIC FUNCTIONS
}

\author{
G. K. HAEUSLEIN
}

Abstract. The purpose of this note is to establish a necessary and sufficient condition for the algebraic independence of certain sets of homogeneous symmetric polynomials which is used in 2 . to solve a problem proposed by L. Flatto [2].

1. We are concerned with the algebraic independence of a set of homogeneous symmetric polynomials in $n$ variables with complex coefficients:

$$
h_{1}, h_{2}, \cdots, h_{n} \in C\left[x_{1}, x_{2}, \cdots, x_{n}\right],
$$

where $h_{k}$ is homogeneous of degree $k$. By the fundamental theorem on symmetric polynomials each $h_{k}$ can be written uniquely as a polynomial in the elementary symmetric polynomials $s_{1}, s_{2}, \cdots, s_{n}$ of $x_{1}, \cdots, x_{n}$. Since $h_{k}$ is homogeneous of degree $k$ it can even be written as a polynomial in $s_{1}, s_{2}, \cdots, s_{k}$ which is linear in $s_{k}$ :

$$
h_{k}=G_{k}\left(s_{1}, s_{2}, \cdots, s_{k-1}\right)+c_{k} \cdot s_{k} \text {, }
$$

where $G_{k}(0,0, \cdots, 0)=0$ and $c_{k} \in C . h_{1}, h_{2}, \cdots, h_{n}$ are algebraically independent over $C$ if and only if each $c_{k} \neq 0$ for $k=1,2, \cdots, n$. Indeed if each $c_{k} \neq 0$ the system (1) can be solved recursively for $s_{1}, s_{2}, \cdots, s_{n}$ in terms of the $h$ s, and $s_{1}, s_{2}, \cdots, s_{n}$ are algebraically independent over $C$. If not all $c_{k} \neq 0$ let $c_{i}$ be the first vanishing $c$. For $i=1$ we have $h_{1}=0$. For $i>1$ we can solve the partial system $h_{k}=G_{k}\left(s_{1}, s_{2}, \cdots, s_{k-1}\right)+c_{k} s_{k}, k=1,2, \cdots, i-1$ for $s_{1}, s_{2}, \cdots$, $s_{i-1}$ in terms of $h_{1}, h_{2}, \cdots, h_{i-1}$ and enter these solutions into $h_{i}$ $=G_{i}\left(s_{1}, s_{2}, \cdots, s_{i-1}\right)$.

It is possible to determine the coefficients $c_{k}$ without knowing the actual representation (1). For $k \leqq n$ let $\omega_{k}$ denote a primitive $k$ th root of unity. Then $\omega_{k}, \omega_{k}^{2}, \cdots, \omega_{k}^{k}$ is the complete set of zeros of the polynomial $x^{k}-1$ and we have

and

$$
\begin{aligned}
s_{i}\left(\omega_{k}, \underset{\omega_{k}}{2}, \cdots, \omega_{k}^{k}, 0, \cdots, 0\right) & =0 \quad \text { for } i<k, \\
s_{k}\left(\omega_{k}, \omega_{k}^{2}, \cdots, \omega_{k}, 0, \cdots, 0\right) & =(-1)^{k-1},
\end{aligned}
$$

Received by the editors August 15, 1969.

A MS Subject Classifications. Primary 1275, 0505. unity.

Key Words and Phrases. Algebraic independence, symmetric polynomials, roots of 


$$
h_{k}\left(\omega_{k}, \underset{\omega_{k}^{2}}{2}, \cdots, \underset{\omega_{k}^{k}}{k}, 0, \cdots, 0\right)=(-1)^{k-1} c_{k} .
$$

We have proved

THEOREM 1. Let $h_{k}$ be a homogeneous symmetric function of degree $k$ in $x_{1}, x_{2}, \cdots, x_{n}$ with complex coefficients $(k=1,2, \cdots, n)$. $h_{1}, h_{2}, \cdots, h_{n}$ are algebraically independent over $C$ if and only if

$$
h_{k}\left(\omega_{k}, \underset{\omega_{k}^{2}}{2} \cdots, \underset{\omega}{\omega_{k}}, 0, \cdots, 0\right) \neq 0
$$

for $k=1,2, \cdots, n$, where $\omega_{k}$ is a primitive kth root of unity.

2. We apply Theorem 1 to the solution of a problem of L. Flatto [2] and prove

Theorem 2. Let $P_{2 k}(x)=\sum_{ \pm}\left( \pm x_{1} \pm x_{2} \pm \cdots \pm x_{n}\right)^{2 k} . \quad P_{2}(x)$, $P_{4}(x), \cdots, P_{2 n}(x)$ are algebraically independent.

Direct computation gives

$$
P_{2 k}(x)=2 n \sum_{\lambda_{1}+\lambda_{2}+\cdots+\lambda_{n}=k} \frac{(2 k) !}{\left(2 \lambda_{1}\right) !\left(2 \lambda_{2}\right) ! \cdots\left(2 \lambda_{n}\right) !} x_{1}^{2 \lambda_{1}} x_{2}^{2 \lambda_{2}} \cdots x_{n}^{2 \lambda_{n}} .
$$

Let $y_{i}=x_{i}^{2}$ for $i=1,2, \cdots, n$. Then $P_{2 k}(x)=h_{k}(y)$ is a homogeneous symmetric function of degree $k$ in $y_{1}, \cdots, y_{n}$. According to Theorem 1 we must show

$$
\sum_{\lambda_{1}+\lambda_{2}+\cdots+\lambda_{k}=k} \frac{1}{\left(2 \lambda_{1}\right) !\left(2 \lambda_{2}\right) ! \cdots\left(2 \lambda_{k}\right) !} \omega_{k}^{\lambda_{1}+2 \lambda_{2}+\cdots+k \lambda_{k}} \neq 0 .
$$

We call the $k$-tuple $\left(\lambda_{1}, \lambda_{2}, \cdots, \lambda_{k}\right)$ a vector of exponents. The "length" of a vector of exponents be the number of its nonzero components. Let $\left\{\nu_{1}, \nu_{2}, \cdots, \nu_{r}\right\}$ be a set of $r \leqq k$ positive integers satisfying $\sum \nu_{i}=k$. We determine all vectors of exponents of length $r$ obtained by assigning $\nu_{1}, \nu_{2}, \cdots, \nu_{r}$ to $r$ different components and compute the sum $S_{k}\left(\nu_{1}, \nu_{2}, \cdots, \nu_{r}\right)$ of the corresponding powers of $\omega_{k}$ : $S_{k}\left(\nu_{1}, \nu_{2}, \cdots, \nu_{r}\right)$

$$
\begin{aligned}
& =\sum_{m_{1}=1}^{k} \omega_{k}^{m_{1} \nu_{1}} \cdot \sum_{m_{2}=1}^{k}\left(1-\delta_{m_{1} m_{2}}\right) \omega_{k}^{m_{2} \nu_{2}} \cdots \cdots \sum_{m_{r}=1}^{k} \prod_{i=1}^{r-1}\left(1-\delta_{m_{i} m_{r}}\right) \omega_{k}^{m_{r} \nu_{r}} \\
& =\sum_{m_{1}, m_{2}, \cdots, m_{r}}^{k} \prod_{i<j}\left(1-\delta_{m_{i} m_{j}}\right) \omega_{k}^{m_{1} \nu_{1}+m_{2} \nu_{2}+\cdots+m_{r} \nu_{r}}=(-1)^{r-1} k(r-1) !
\end{aligned}
$$

This equation is clearly true for $r=1, \nu_{1}=k$. Assume now that $r>1$. Since $\nu_{r}<k$ we have $\sum_{m_{r}} \omega_{k}^{m_{r} \nu_{r}}=0$ and $\sum_{m_{r}} \prod_{i<r}\left(1-\delta_{m_{i} m_{r}}\right) \omega_{k}^{m_{r} \nu_{r}}$ $=-\sum_{i<r} \omega_{k}^{m_{i} \nu_{r}}$ giving 


$$
\begin{aligned}
S_{k}\left(\nu_{1}, \cdots, \nu_{r}\right) & =-\sum_{l=1}^{r-1} S_{k}\left(\nu_{1}+\delta_{1} \nu_{r}, \nu_{2}+\delta_{2} l \nu_{r}, \cdots, \nu_{r-1}+\delta_{r-1, l} \nu_{r}\right) \\
& =(-1)(r-1) \cdot(-1)^{r-2} \cdot k(r-2) !
\end{aligned}
$$

by induction.

To obtain the sum of all terms in (2) which have the common coefficient $\left(\left(2 \nu_{1}\right) ! \cdots\left(2 \nu_{r}\right) !\right)^{-1}$ we must divide $S_{k}\left(\nu_{1}, \nu_{2}, \cdots, \nu_{r}\right)$ by $n_{1} ! n_{2} ! \cdots n_{k}$ ! where $n_{i}$ is the number of times the integer $i$ occurs in the set $\left\{\nu_{1}, \nu_{2}, \cdots, \nu_{r}\right\}$. Using

$$
\sum_{l=1}^{k} n_{l}=r
$$

we get

$$
\begin{aligned}
& \sum_{\lambda_{1}+\lambda_{2}+\cdots+\lambda_{k}=k} \frac{1}{\left(2 \lambda_{1}\right) ! \cdots\left(2 \lambda_{k}\right) !} \omega^{\lambda_{1}+2 \lambda_{2}+\cdots+k \lambda_{k}} \\
= & \sum_{\Sigma l_{n}=k}(-1)^{\left(\sum n_{l}-1\right)} k \frac{\left(\sum n_{l}-1\right) !}{n_{1} ! n_{2} ! \cdots n_{k} !} \cdot \frac{1}{(2 !)^{n_{1}}(4 !)^{n_{2}} \cdots(2 k) ! n_{k}} .
\end{aligned}
$$

Now we apply to the right-hand side of (3) the following result of Cauchy [1]: The formal equation

$$
b_{1} z+\frac{1}{2} b_{2} z^{2}+\frac{1}{3} b_{3} z^{3}+\cdots=\log \left(1+a_{1} z+a_{2} z^{2}+\cdots\right)
$$

gives rise to the identity

$$
b_{k}=k \sum_{\Sigma l_{l}=k}(-1)^{\Sigma n_{l}-1} \frac{\left(\sum n_{l}-1\right) !}{n_{1} ! n_{2} ! \cdots n_{k} !} a_{1}^{n_{1} a_{2} n_{2}} \cdots a_{k}^{n_{k}} .
$$

By letting $a_{l}=1 /(2 l !)$ for $l=1,2, \cdots, k$ we obtain the right-hand side of (3). It is convenient to replace in (4) the variable $z$ by $t^{2}$ and to differentiate with respect to $t$. Then

$$
\sum_{l=0}^{\infty} \frac{z^{l}}{2 l !}=\sum_{l=0}^{\infty} \frac{t^{2 l}}{2 l !} \stackrel{3}{=} \frac{1}{2}\left(e^{t}+e^{-t}\right)
$$

and we have

$$
\sum_{i=1}^{\infty} 2 b_{i} t^{2 i-1}=\frac{d}{d t} \log \left(\frac{e^{t}+e^{-t}}{2}\right)=\tanh (t) .
$$

We obtain the value of (2) by dividing 2 into the $(2 k-1)$ st coefficient of the Taylor expansion of $\tanh (t)$. According to [3, pp. 298299], this coefficient equals 


$$
\frac{2^{2 k}\left(2^{2 k}-1\right) B_{2 k}}{(2 k) !}, \quad B_{2 k}=(-1)^{k-1} \frac{2(2 k) !}{(2 \pi)^{2 k}} \sum_{l=1}^{\infty} \frac{1}{l^{2 k}}
$$

and is always different from zero. This establishes the theorem.

\section{BIBLIOGRAPHY}

1. A. Cauchy, Mémoire sur diverses formules relatives d l'algèbre et da la théorie des nombres, C. R. Acad. Sci. Paris 12 (1841), 698-711.

2. L. Flatto, Basic sets of invariants for finite reflection groups, Bull. Amer. Math. Soc. 74 (1968), 730-734. MR 37 \#1483.

3. T. J. L. Bromwich, An introduction to the theory of infinite series, 2nd ed., Macmillan, London, 1926.

OAK Ridge National Laboratory, ${ }^{1}$ OAK Ridge, Tennessee 37830

1 Operated by Union Carbide Corporation for the U. S. Atomic Energy Commission. 